\title{
Pensar en género en el Ejército Nacional: reflexiones y escenarios de abordaje en la institución ${ }^{1}$

https://doi.org/10.21830/9789585318304.11

\author{
Leidy Johana Cabrera Cabrera² \\ Escuela Militar de Cadetes "General José María Córdova" \\ Maria Antonieta Corcione-Nieto ${ }^{3}$ \\ Escuela de Aviación del Ejército
}

\section{Resumen}

Este capítulo tiene por objeto presentar un análisis de los diez estudios precedentes compilados en esta obra, como aportes conceptuales y escenarios de abordaje sobre la mujer militar en el Ejército Nacional de Colombia. A partir de una revisión de los textos, se identificaron varios ejes de reflexión en cuanto al contexto militar dispuesto para las mujeres y las necesidades específicas a futuro en el campo de los estudios de género.

Palabras clave: Ejército Nacional de Colombia; formación militar; género; identidades; mujer militar

\section{Introducción}

En los últimos años, los estudios sobre la mujer militar en el Ejército Nacional de Colombia han sido objeto de un interés especial por parte de Escuela Militar de Cadetes “General José María Córdova” (ESMIC), en consonancia con una tendencia

1 Este capítulo presenta de forma conjunta los resultados de dos proyectos de investigación: "Diplomado virtual en género para la transformación del Ejército Nacional" y "Enfoques diferenciales en el Ejército Nacional: una propuesta desde las ciencias militares" del Grupo de Investigación en Ciencias Militares, de la Escuela Militar de Cadetes "General José María Córdova" (ESMIC), categorizado en B por Minciencias y con código de registro COL0082556. Los puntos de vista y los resultados de este artículo pertenecen a las autoras y no reflejan necesariamente los de las instituciones participantes.

2 Magíster y especialista en Docencia e Investigación Universitaria (Universidad Sergio Arboleda). Trabajadora Social (Universidad de La Salle). Docente e investigadora en ciencias sociales y humanas. Asesora de investigación del Observatorio de Género de la Escuela Militar de Cadetes “General José María Córdova”. Orcid: https://orcid.org/0000-0002-6398-6933 - Contacto: leidy.cabrerats@esmic.edu.co

$3 \mathrm{PhD}$ en Antropología (Universidad de los Andes). Magíster en Biología Humana (Universidad Autónoma de Barcelona). Líder del Grupo de Investigación en Ciencias Militares de la Escuela Militar de Cadetes “General José María Córdova”. Orcid: https://orcid.org/0000-0002-3509-5519 - Contacto: mariacorcionenieto@cedoc.edu.co 
general nacional de incremento en la diversidad de temáticas, en las metodologías aplicadas y en los marcos teóricos elegidos (Rodríguez Pizarro \& Ibarra Melo, 2013). Los temas de investigación han abordado una amplia gama de preocupaciones e intereses, como la caracterización de las mujeres cadetes vinculadas a la ESMIC (Camacho, 2014; Fernández et al., 2018), las dimensiones del liderazgo militar que ellas ejercen (Perilla Toro \& Cabrera-Cabrera, 2018) y una propuesta de ajustes a los procesos de formación con enfoque de género (Fernández-Osorio \& Latorre Rojas, 2018), entre otros. Estas perspectivas marcan un avance investigativo que ubica el género como un eje de estudio determinante en el proceso de evolución que vive constantemente la institución.

Por definición, diversos autores describen el género como una construcción social y simbólica, cuya perspectiva apunta hacia la distinción entre la diferencia sexual y los roles sociales que se construyen a partir de dicha diferencia (Miranda, 2012, p. 346). Así, en esta obra se retoman cinco escenarios diferentes de análisis sobre la mujer militar, los cuales enriquecen la comprensión de sus roles de estudiantes, oficiales y suboficiales de carrera y personal administrativo en el Ejército Nacional. Cada capítulo dispone de un eje central: i) identidades femeninas, ii) motivaciones para el ingreso de mujeres de arma, iii) lineamientos de ley para mujeres en estado de embarazo, iv) inclusión de competencias de igualdad en la formación y v) historias de vida.

En este sentido, los escenarios desarrollados mantienen una tendencia hacia diferentes líneas de conocimiento que definen un abordaje de género en el contexto militar para comprender mejor las prácticas sociales que se dan en su interior. Dicha tendencia marca un horizonte de sentido para cada capítulo de acuerdo con las investigaciones que se adelantan en el Ejército Nacional. Así, se asumen como representativos los resultados que se exponen en cada uno de ellos y son tomados como objeto de estudio para el análisis que se presenta a continuación.

\section{Método}

Actualmente la minería de textos se utiliza para identificar tendencias, desviaciones y asociaciones a partir de la analítica de datos de información textual (Universidad de La Salle, 2020). En este sentido, para analizar la información de los capítulos precedentes y ofrecer una visión objetiva de sus contenidos se implementaron dos técnicas para visualizar la información textual: nube de palabras y diagramas de frecuencia de palabras. 
La nube de palabras, entendida como la representación visual de las palabras contenidas en un texto en donde las frecuencias de los términos están marcadas por el tamaño de la letra de las palabras (Martos, 2014), permite identificar la jerarquización e importancia de cada término identificado. Esta técnica se implementó de forma general para cada uno de los capítulos del libro. Para poderla desarrollar, se eliminaron los espacios, números, preposiciones, artículos y conectores del texto general de los capítulos precedentes (Universidad de La Salle, 2020), y los datos de la matriz resultante se procesaron con el programa Atlas-Ti. Dada la gran cantidad de palabras de la nube resultante, se optó por visualizar solo los 70 términos de mayor frecuencia.

Los diagramas de frecuencia de palabras, por su parte, son formas alternativas de presentar la nube de palabras (Universidad de La Salle, 2020). Esta técnica se implementó para determinar la frecuencia de términos en todos los capítulos, tanto de forma individual como en su conjunto. Para cada capítulo, se tomaron también los 70 términos más frecuentes y se presentaron en una gráfica de frecuencias acumuladas para determinar su peso relativo. El diagrama de frecuencias de todos los capítulos precedentes en su conjunto incluyó los 25 términos más frecuentes, así como su distribución porcentual por capítulo.

A partir de estos resultados, se establecieron las categorías que determinan las tendencias del libro y permiten generar las perspectivas para investigaciones futuras en el campo de género desde el ámbito militar.

\section{Resultados}

Para describir las frecuencias de palabras, se mencionarán solamente aquellas que se ubican dentro del $50 \%$ de frecuencia acumulada en cada capítulo. Así, para el capítulo 1, "De-construyendo identidades femeninas en el Ejército Nacional de Colombia: las mujeres militares", ocho palabras presentaron la mayor frecuencia: mujeres, identidad, militar, género, militares, femenino, mujer y social, y ocuparon el $49,2 \%$ de las palabras más frecuentes en este capítulo (figura 1a). La muestra total de las 70 palabras más frecuentes corresponde al 10,6\% de las palabras de todo el capítulo (figura 2).

En el capítulo 2, "Mujeres de arma: motivaciones para el ingreso al Ejército Nacional de Colombia", doce palabras tuvieron la mayor frecuencia. Estas fueron militar, mujeres, género, oficiales, militares, arma, Ejército, vida, carrera, escuela, profesional y armas, y ocuparon el 49,9\% de las palabras más frecuentes en el capítulo (figura 1b). La muestra total de las 70 palabras más frecuentes corresponde al 9,3\% de las palabras de todo el capítulo (figura 2). 


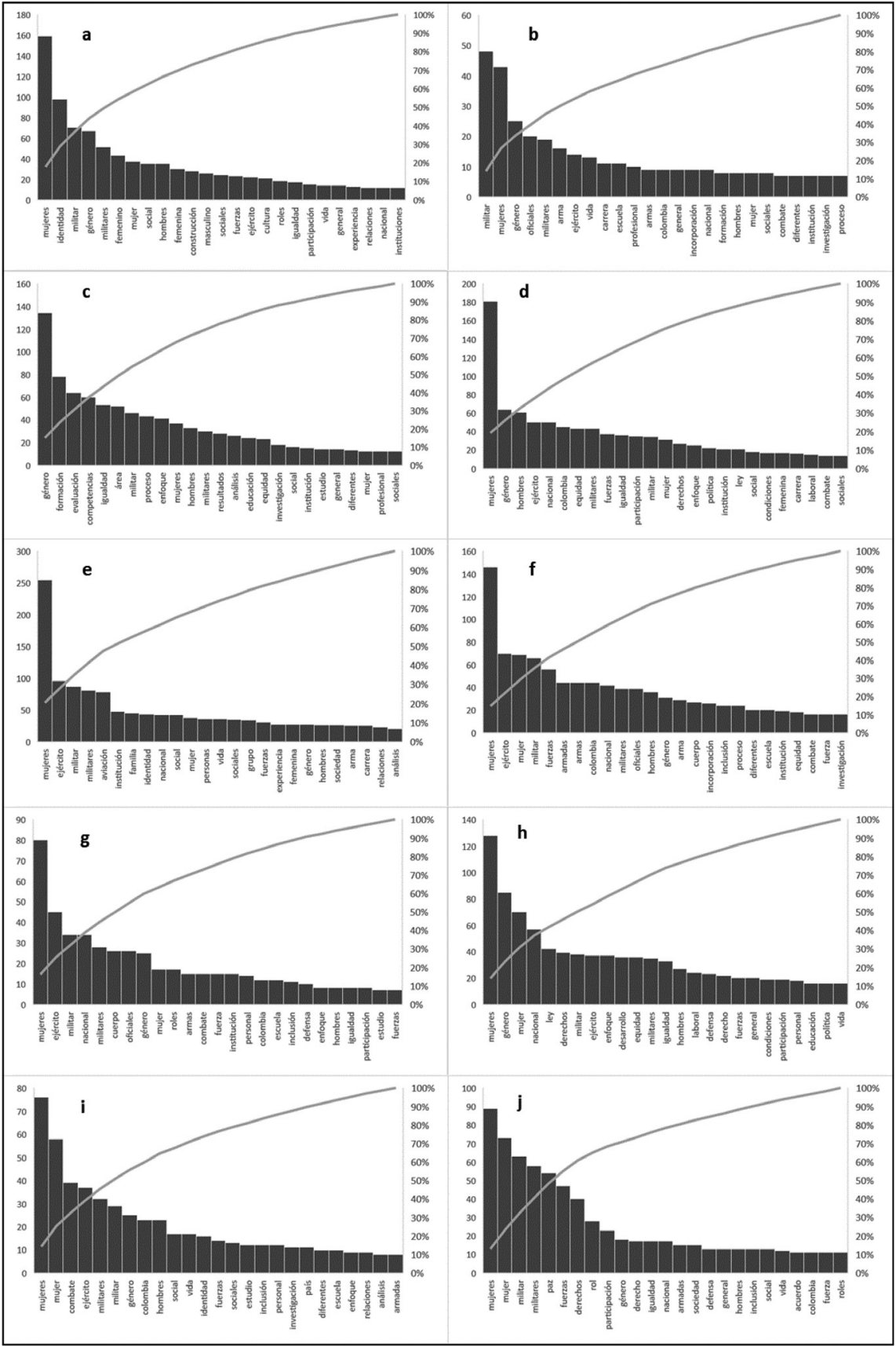

Figura 1. Diagrama de frecuencias de cada uno de los capítulos: a) capítulo 1; b) capítulo 2; c) capítulo 3; d) capítulo 4; e) capítulo 5; f) capítulo 5; g) capítulo 6; h) capítulo 7; i) capítulo 8; j) capítulo 9 y k) capítulo 10 .

Fuente: Elaboración propia 
En el capítulo 3, "Transversalización: evaluación de competencias de igualdad de género en la formación de las mujeres militares", ocho palabras tuvieron la mayor frecuencia. Estas fueron género, formación, evaluación, competencias, igualdad, área, militar y proceso, y ocuparon el 49,0\% de las palabras más frecuentes en este capítulo (figura 1c). La muestra total de las 70 palabras más frecuentes corresponde al $11,2 \%$ de las palabras de todo el capítulo (figura 2).

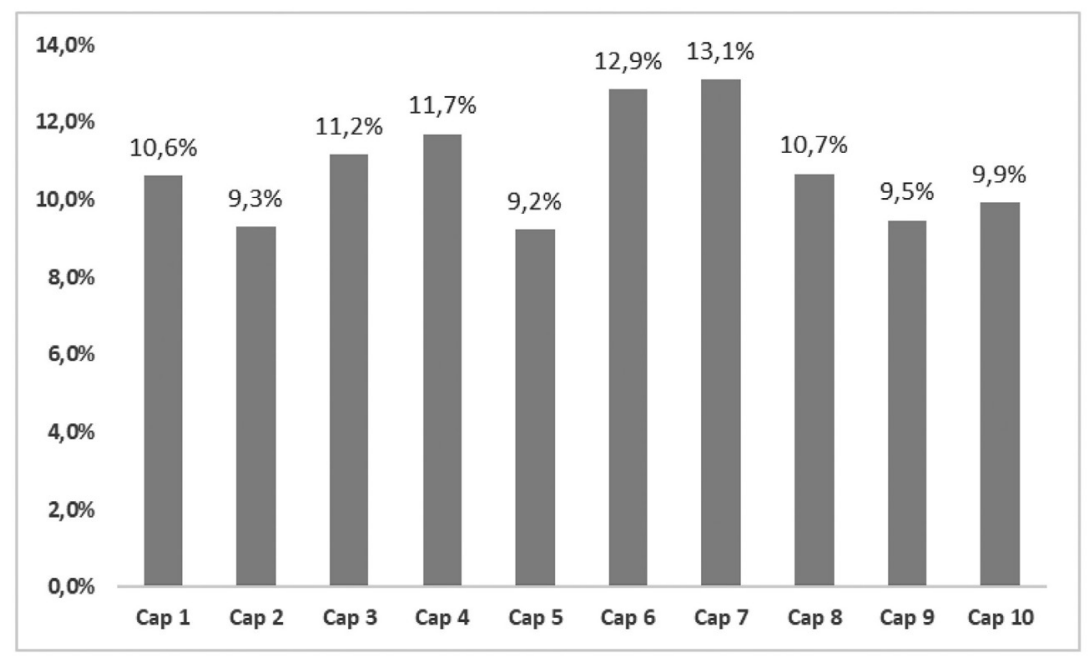

Figura 2. Porcentaje del texto cubierto por las 70 palabras con mayor frecuencia del libro en cada capítulo.

Fuente: Elaboración propia

En el capítulo 4, "Trato justo y equitativo con enfoque diferencial en el Ejército Nacional de Colombia: análisis de la normatividad vigente", fueron ocho las palabras con mayor frecuencia: mujeres, género, hombres, Ejército, Colombia, equidad, militares y fuerzas, y ocuparon el 48,6\% de las palabras más frecuentes en este capítulo (figura 1d). La muestra total de las 70 palabras más frecuentes corresponde al $11,7 \%$ de las palabras de todo el capítulo (figura 2).

En el capítulo 5, "Mujeres de Aviación del Ejército Nacional: desligándose de las imágenes estereotipadas", diez palabras tuvieron la mayor frecuencia. Estas fueron mujeres, Ejército, militar, militares, aviación, institución, familia, identidad, nacional y social, y ocuparon el 49,6\% de las palabras más frecuentes en este capítulo (figura 1e). La muestra total de las 70 palabras más frecuentes corresponde al 9,2\% de las palabras de todo el capítulo (figura 2).

En el capítulo 6, "El arma de Caballería del Ejército Nacional de Colombia: avances en la inclusión de mujeres", once palabras tuvieron la mayor frecuencia: 
mujeres, Ejército, mujer, militar, fuerzas, armadas, armas, Colombia, Nacional, militares y oficiales, y ocuparon el 49,8\% de las palabras más frecuentes en este capítulo (figura 1f). La muestra total de las 70 palabras más frecuentes corresponde al $12,9 \%$ de las palabras de todo el capítulo (figura 2).

En el capítulo 7, "El quehacer de las mujeres oficiales del cuerpo logístico en el Ejército Nacional de Colombia", nueve palabras tuvieron la mayor frecuencia. Estas fueron mujeres, Ejército, Militar, Nacional, militares, cuerpo, oficiales, género y mujer, y ocuparon el 50,7\% de las palabras más frecuentes en este capítulo (figura 1g). La muestra total de las 70 palabras más frecuentes corresponde al 13,1\% de las palabras de todo el capítulo (figura 2).

En el capítulo 8, "Lineamientos para los cursos de ley para las mujeres en embarazo o lactantes del Ejército Nacional: propuesta desde un enfoque diferencial”, once palabras tuvieron la mayor frecuencia: mujeres, género, mujer, Nacional, ley, derechos, Militar, Ejército, enfoque, desarrollo y equidad, y ocuparon el 50,8\% de las palabras más frecuentes en este capítulo (figura 1h). La muestra total de las 70 palabras más frecuentes corresponde al 10,7\% de las palabras de todo el capítulo (figura 2).

En el capítulo 9, "Historias de vida de mujeres militares en Colombia y Estados Unidos: un análisis reflexivo", nueve palabras tuvieron la mayor frecuencia: mujer, combate, Ejército, militares, Militar, género, Colombia, hombres y social, y ocuparon el 50,4\% de las palabras más frecuentes en este capítulo (figura 1i). La muestra total de las 70 palabras más frecuentes corresponde al $9,5 \%$ de las palabras de todo el capítulo (figura 2).

Por último, en el capítulo 10, "El rol de la mujer militar y su aporte a la sociedad en el posacuerdo de paz", siete palabras tuvieron la mayor frecuencia. Estas fueron mujeres, mujer, militar, militares, paz, fuerzas y derechos, y ocuparon el $48,3 \%$ de las palabras más frecuentes en este capítulo (figura $1 \mathrm{j}$ ). La muestra total de las 70 palabras más frecuentes corresponde al 9,9\% de las palabras de todo el capítulo (figura 2).

\section{En general: palabras más mencionadas}

En general, las 70 palabras más frecuentes del libro tuvieron un promedio de presencia de $10,8 \%$ de todo el texto de cada capítulo. El número de estas palabras frecuentes por capítulo fue en promedio de 9,4, mientras que el volumen de palabras válidas —entendidas como aquellas que permiten realizar inferencias dentro del texto- en cada capítulo fue en promedio de 9.597 y las palabras frecuentes se repiten en promedio 14,4 veces en cada capítulo. Así, estas palabras determinan tanto el contexto general de la intencionalidad del texto como el horizonte de sentido de cada capítulo. No obstante, es importante ponderar el conjunto total de palabras de acuerdo con los textos de los diez capítulos en conjunto. 
Las 25 palabras más frecuentes de los últimos cinco capítulos fueron mujeres (1.194) ${ }^{4}$, militar (515), género (501), militares (416), mujer (413), Ejército (383), nacional (277), hombres (270), fuerzas (246), igualdad (198), Colombia (184), identidad (170), social (168), equidad (154), institución (151), participación (143), derechos (134), enfoque (134), oficiales (133), formación (130), vida (130), proceso (127), sociales (127), femenino (117) y armas (115). De la misma manera, estas 25 palabras son mencionadas en promedio 261 veces a lo largo de cada uno de los diez capítulos (figura 3) y ponen de manifiesto el mensaje general del libro; sobre ellas se realizará un análisis conceptual más adelante.

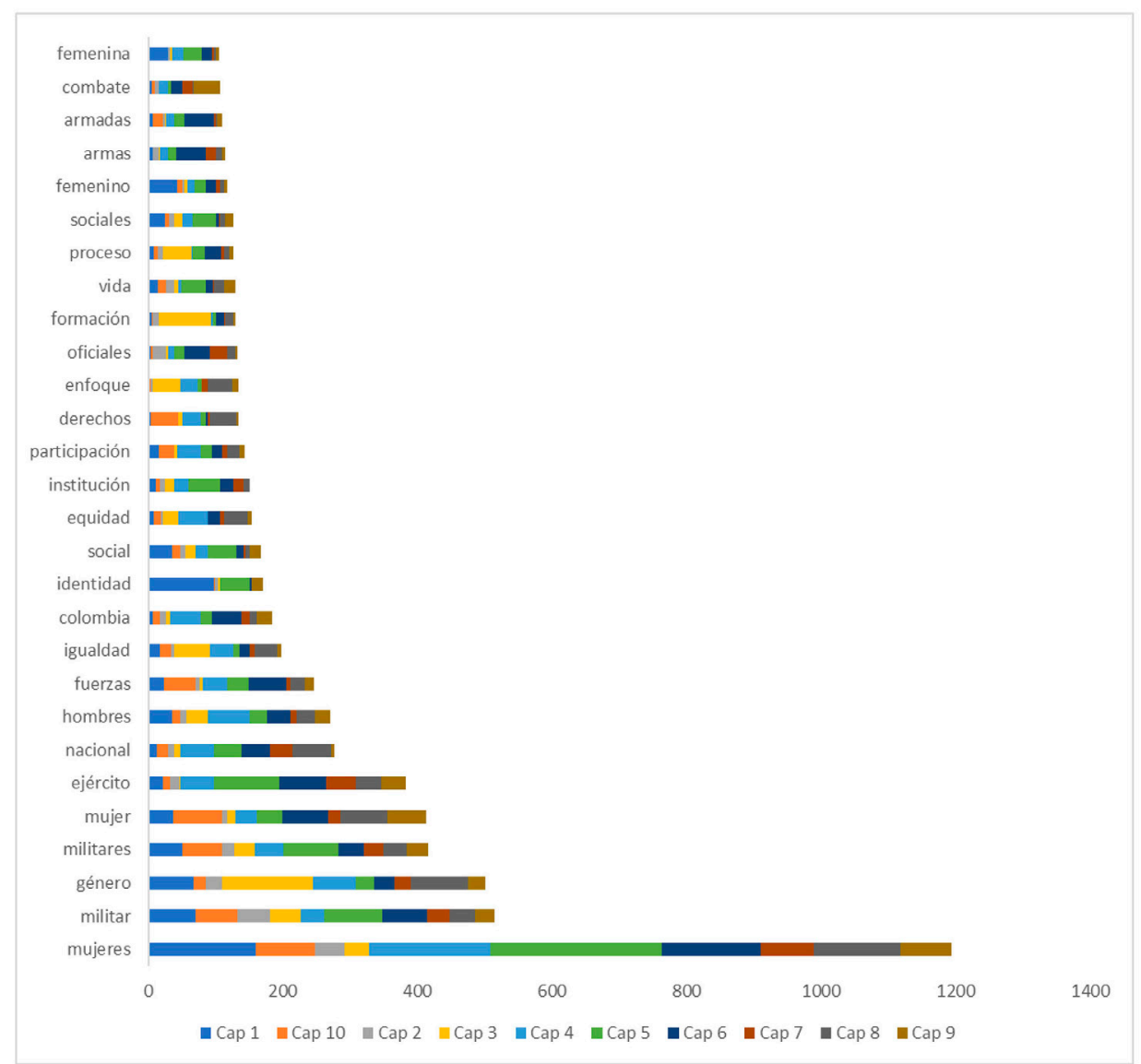

Figura 3. Diagrama de frecuencias de las principales palabras de los diez capítulos. Se resalta la proporción en la que cada capítulo aporta a la frecuencia.

Fuente: Elaboración propia

4 Se muestra entre paréntesis la frecuencia absoluta de la palabra. 


\section{Horizonte de sentido: el mensaje central}

Las 70 palabras que más se mencionan se presentan según su número en un esquema de nube de palabras (figura 4) que deja ver el sentido general de los estudios adelantados y los nuevos análisis que pueden consolidarse en futuras fases de desarrollo.

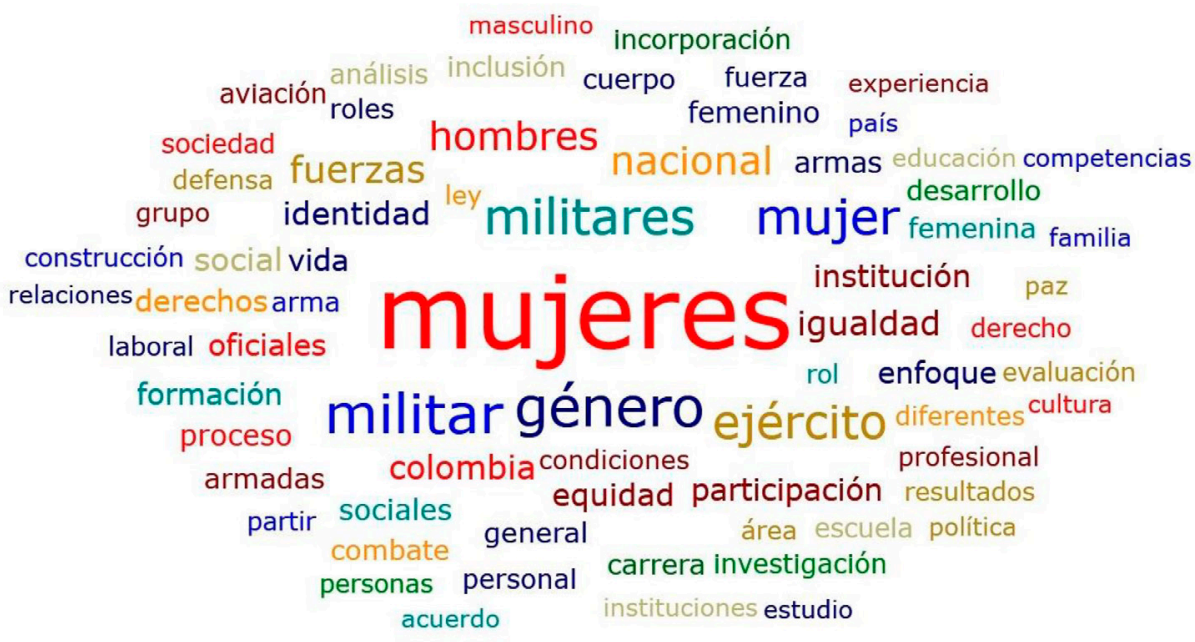

Figura 4. Marca de nube de los documentos revisados

Fuente: Elaboración propia a partir del programa Atlas-Ti

Es importante resaltar que la palabra mujeres fue la más abundante en ocho capítulos, mientras que las palabras militar y género fueron las más abundantes en solo un capítulo cada una de ellas. Considerando las dos palabras más numerosas por cada capítulo se obtiene que las palabras más utilizadas fueron mujer, Ejército, identidad y formación. Para el análisis se abordaron, en especial, estas primeras palabras en su escenario de desarrollo, a partir de las cuales se generaron dos líneas complementarias de estudio que, de acuerdo con los aportes de este libro, señalan nuevos escenarios.

\section{Entre lo singular y lo plural: mujer, mujeres / militar, militares}

La consolidación de un abordaje singular y plural sobre los términos mujer y militar se debe a las identidades que se construyen en el contexto militar, que 
son justamente un objeto de estudio de gran relevancia para las ciencias sociales y humanas (De la Torre, 2007; Mercado y Hernández, 2010; Snow, 2001).

Pensar en una identidad implica pensar en la relación con los otros desde lo excluyente, por lo cual aquella se convierte en una construcción social desde la diferencia. Sin embargo, es necesario hablar de múltiples identidades que interactúan entre sí, en diferentes momentos y lugares, desde la imaginación, con el cuerpo y con las emociones de los individuos (Harré \& Daviés, 1990). Snow (2001) indica que existen tres tipos distintos de identidad: las identidades personales, las identidades sociales y las identidades colectivas, que se configuran a lo largo de la vida de los individuos de manera sinérgica en espacio y tiempo.

Para el caso de las cuatro palabras enunciadas en este apartado (mujer/mujeres y militar/militares), y de acuerdo con lo planteado anteriormente, se podría decir que el abordaje de los conceptos de mujer y militar corresponde a una construcción de identidad individual y una construcción social. Las identidades personales o individuales son los atributos y significados que el actor se atribuye a sí mismo, son autodesignaciones y atribuciones personales consideradas distintivas del y por el individuo. Las identidades personales pueden derivarse de la titularidad de roles o membresías basadas en categorías, pero no son necesariamente comparables desde la importancia relativa de los roles sociales o membresía de categoría (Snow \& Corrigall-Brown, 2015).

Por su parte, las identidades sociales son las identidades atribuidas o imputadas a otros en un intento de situarlos en el espacio social. Ellos se basan típicamente en roles sociales establecidos, como categorías de género o étnicas y nacionales, por lo tanto, a menudo se las denomina "identidades de roles" (Stryker, 1980) o "identidades categóricas” (Calhoun, 1997).

En lo que se refiere al concepto de mujeres, este obedece a una identidad colectiva. Las identidades colectivas tienen su base "en el sentimiento compartido de la existencia de unicidad o un nosotras. Dentro del sentimiento compartido de la existencia de un nosotras, se constituye un sentimiento de agenciamiento colectivo, como componente de acción de las identidades" (Snow, 2001, p. 3).

El concepto de militares se ubica de manera trasversal en cada uno de estos escenarios como una identidad social. Así, el concepto mujer(es) militar(es) implica una serie de significados simbólicos que permiten que las identidades colectivas, sociales e individuales sean expresadas y afirmadas (Snow, 2001) en sus diferentes roles. Lo anterior refleja la importancia de identificar una visión compartida de las características y valores distintivos propios de las mujeres militares del Ejército Nacional como organización. 
En efecto, la comprensión de estos conceptos puede abordarse así: los conceptos de mujer y de mujeres militares implican mantener un reconocimiento de las distintas formas de identidad que ellas expresan y que pueden verse en la cotidianidad militar, familiar, social y cultural, así como en aspectos más profundos de la construcción de su yo.

\section{Hacia una cultura de género en las Fuerzas Militares}

Superada la visión reduccionista de atribuir a las mujeres militares (administrativas y de arma) un único rol, los estudios aquí presentados muestran nuevas perspectivas de abordaje en las que se reconocen las experiencias individuales y colectivas femeninas sobre la profesión militar: es desde su propia voz que se expresa la necesidad de resignificar sus roles para que avance la transformación del Ejército Nacional en ese sentido.

\section{Formación militar: una herencia patriarcal en renovación}

El escenario militar, por tradición, ha favorecido el desarrollo de un liderazgo masculino. Estudios como el de García y López (2006), realizados en organizaciones militares, confirman que el éxito en el liderazgo suele depender de su conformación bajo un modelo masculino (Boldry et al., 2001; Lupano et al., 2008). Sin embargo, esta perspectiva ha tenido fuertes críticas, por no integrar en sus análisis los alcances de las estrategias de liderazgo y desarrollo organizacional que abanderan las mujeres y que cada vez toman más fuerza pese a su baja representatividad como fuerza laboral (González \& Camacaro, 2014).

La incongruencia en roles de liderazgo para el caso de las mujeres se acentúa en ambientes culturalmente masculinos como las organizaciones militares. En estas, son escasos los referentes femeninos de liderazgo, lo cual puede generar incertidumbres en la formación de cadetes mujeres porque la identificación con modelos masculinos se perpetúa (Perilla \& Cabrera, 2018).

La formación militar, por su naturaleza, ha sido definida desde sus inicios a partir de un enfoque patriarcal, basado en una reconstrucción de los roles de género que emula la división masculino-femenino de la sociedad (Castañeda, 2008). Esta condición fue poco estudiada, debido a la predominante masculinidad de las Fuerzas Militares. Actualmente, los estudios en este campo han aumentado 
(Corcione Nieto \& Cabrera Cabrera, 2018) y sus análisis han superado la perspectiva patriarcal tradicional de la formación militar. De hecho, en algunos de estos estudios, los roles de las mujeres militares marcan otras líneas de comprensión sobre un saber hacer en contexto militar.

Es bien sabido que la formación militar es un objeto de estudio determinante en el avance hacia la evolución institucional. Sin embargo, estos estudios tienen mayor pertinencia cuando trascienden la descripción de los modelos existentes inscritos en las relaciones de poder hombre-mujer establecidas, ya que apuntan hacia el reconocimiento equitativo de las experiencias de hombres y mujeres que ejercen su quehacer militar. Lo anterior es clave para una formación integral, ajustada a las realidades institucionales y sociales del país.

\section{Tareas pendientes}

Sin duda, el campo de conocimiento en estudios de género, en especial, en cuanto a mujeres militares, es una tarea que va en avance, y que aún tiene varios componentes por abordar, dada su complejidad. Los diferentes estudios presentados aquí han mostrado algunos escenarios, pero corresponde a un compromiso científico poner de manifiesto preguntas abiertas que resultan de este ejercicio y que se convierten, a su vez, en tareas pendientes para futuras investigaciones:

- ¿Cómo se configura la fundamentación del enfoque de género en el contexto militar?

- ¿Cuáles son los alcances del enfoque de género en el proceso formativo de militares?

- ¿Cómo están las demás fuerzas del Ejército Nacional en relación con los roles desempeñados por las mujeres?

- ¿Cuáles son los determinantes identitarios y dinámicas en el contexto militar femenino?

- ¿Los lineamientos normativos actuales son suficientes para un abordaje trasversal del enfoque de género?

- ¿Cuál es la visión compartida de las características y valores distintivos propios de la mujer militar en el Ejército Nacional como organización?

Los hombres y las mujeres están vinculados a una herencia de modelos de género y los estudios prospectivos sobre el avance en esto puede definir hitos que marcan nuevos escenarios y abordajes desde la investigación social. En la actualidad, 
las relaciones de género en una institución como el Ejército Nacional de Colombia marcan transformaciones que influyen en la comprensión de sus nuevas realidades y que muestran cambios complejos en la práctica militar y cotidiana asumida por hombres y mujeres. Cómo se dan estas relaciones, qué estrategias se desarrollan y cuáles son los efectos políticos son algunos de los interrogantes que, desde la observación de los fenómenos, pueden definir un horizonte de sentido importante para estructurar el enfoque de género en el Ejército Nacional de Colombia.

\section{Referencias}

Boldry, J., Wood, W., \& Kashy, D. (2001). Gender stereotypes and the evaluation of men and women in military training. Journal of Social Issues, 57(4), 689-705.

Calhoun, C. (1997). Nationalism. University of Minnesota Press.

Camacho, C. (2014). Ejército, feminidades y géneros performativos: las experiencias de ser mujer y militar en la Escuela Militar de Cadetes “General José María Córdova” [Tesis de maestría]. Pontificia Universidad Javeriana.

Castañeda, A. (2008). El Ejército, ¿el reflejo más bello del modelo patriarcal? En C. Torres del Río, \& S. Rodríguez (Ed.), De Milicias reales a militares contrainsurgentes. La Institución militar en Colombia del siglo XVIII al XXI. Editorial Pontificia Universidad Javeriana.

Corcione Nieto, M. \& Cabrera Cabrera, L. (2018). Identidad e ideología. Dinámicas culturales entre los estudiantes de la Escuela Militar de Cadetes. En A. Fernández Osorio, \& E. Latorre Rojas (Eds.), La construcción del rol de la mujer militar (pp. 41-65). Sello Editorial ESMIC.

De la Torre, C. (2007). Identidad, identidades y ciencias sociales contemporáneas: conceptos, debates y retos [Conferencia]. Universidad Católica de Oriente. https:/letrajoven.wordpress.com/2011/09/02/ identidad-identidades-y-ciencias-sociales-contemporaneas-conceptos-debates-y-retos/

Fernández, A., Latorre, E. J., \& Mayorga, N. (2018). The 2018 Colombian Military Academy dataset: A sociological study of population. Revista Cientifica General José María Córdova, 16(23), 147-162. http://dx.doi.org/10.21830/19006586.345

Fernández-Osorio, A., \& Latorre Rojas, E. (2018). Innovación educativa para el fomento de la equidad de género en las Fuerzas Militares. En A. Fernández Osorio, \& E. Latorre Rojas (Eds.), La construcción del rol de la mujer militar (pp. 141-160). Escuela Militar de Cadetes "General José María Córdova”.

García, R., \& López, E. (2006). Congruencia de rol de género y liderazgo: el papel de las atribuciones causales sobre el éxito y el fracaso. Revista Latinoamericana de Psicología, 38(2), 245-257.

González, M. C., \& Camacaro, D. (2014). Incorporación de la perspectiva de género en las organizaciones. Una propuesta estratégica situacional. Parte I. Salud de los Trabajadores, 22(2), 151-154.

Harré, R., \& Davies, B. (1990). Positioning: The discursive production of selves. Journal for the Theorie of Social Behavior. 20(1), 43-63. https://doi.org/10.1111/j.1468-5914.1990.tb00174.x

Lupano, M., Castro Solano, A., \& Casullo, M. (2008). Prototipos de liderazgo masculino y femenino en población militar. Revista de Psicología, 26(2), 195-218. https://doi.org/10.18800/ psico.200802.001 
Martos, G. (2014). Nubes de palabras en R: otra forma del leer el periódico. https://rpubs.com/gabrielmartos/wordcloudnwsppr

Mercado, A., \& Hernández A. (2010). El proceso de construcción de la identidad colectiva. Revista Convergencia, 53, 229-251.

Miranda, M. (2012). Diferencia entre la perspectiva de género y la ideología de género. Díkaion, 21(2), 337-356.

Perilla Toro, L., \& Cabrera-Cabrera, L. (2018). Caracterización del liderazgo militar desde una perspectiva de género. En A. Fernández Osorio, \& E. Latorre Rojas (Eds.), La construcción del rol de la mujer militar (pp. 67-91). Escuela Militar de Cadetes "General José María Córdova”.

Rodríguez Pizarro, A. N. \& Ibarra Melo, M. E. (2013). Los estudios de género en Colombia. Una discusión preliminar. Sociedad y Economía, 24, 15-46.

Snow, D. (2001). Collective identity and expressive forms. University of California.

Snow, D., \& Corrigall-Brown, C. (2015). Collective identity. In International Encyclopedia of the Social \& Behavioral Sciences. https://doi.org/10.1016/B978-0-08-097086-8.10403-9

Stryker, S. (1980). Symbolic interactionism: A structural version. Benjamin/Cummings Publishing Company.

Universidad de La Salle. (2020). Meta-análisis taller DOFA. Oficina de Planeación Estratégica de la Universidad de La Salle. 